\title{
El puerto de La Plata: de miradas locales y regionales hacia un horizonte atlántico
}

The port of La Plata: from local and regional views to an Atlantic horizon

Santiago Prieto

DOI: https://doi.org/10.24215/23468971e116

Universidad Nacional de La Plata, Argentina

prieto.santiago88@gmail.com

Recepción: 30 Abril 2020

Aprobación: 25 Junio 2020

\section{Resumen:}

Este Balance historiográfico presenta los primeros resultados surgidos de nuestra tesis de Licenciatura en Historia. Proponemos un recorrido por la bibliografía que abordó el puerto de La Plata, considerados en virtud de constituir un primer período de análisis (1883-1925), a partir del cual investigamos su historia económica. Planteamos revalorizar este objeto de estudio con el ánimo de darle un nuevo lugar en la historiografía platense al situarlo en el ámbito atlántico desde una nueva perspectiva de análisis. Nos interesa indagar en la complejidad histórica que concierne al puerto, en tanto entramado económico, social, político, que une una región con las dinámicas globales.

Palabras Clave: Puerto, La Plata, Historia, Economía, Atlántico.

\section{Abstract:}

This historiographic balance presents the first results arising from our thesis in History. We propose a review of the bibliography that addressed the port of La Plata, considered by virtue of constituting a first period of analysis (1883-1925), from which we investigated its economic history. We propose to revalue this object of study with the aim of giving it a new place in La Plata historiography by locating it in the Atlantic field from a new perspective of analysis. We are interested in investigating the historical complexity that concerns the port, as an economic, social, and political, framework that unites a region with global dynamics.

KEYWORDS: Port, La Plata, History, Economy, Atlantic.

En el marco de nuestra tesis de Licenciatura nos encontramos abocados a investigar la historia del puerto de la Plata. Hemos delimitado el período comprendido entre el año 1883, cuando se inicia oficialmente su construcción (Ortiz, 1943), hasta el momento en que se instala la destilería de YPF (Scoccia, 1950; de Paula, 1987), lo cual nos permite dar un cierre temporal hacia $1925 .{ }^{1}$ De esta manera, planteamos un estudio de caso en el cual es posible indagar cuatro ejes temáticos que conciernen al desarrollo del capitalismo argentino: la exportación de productos agrarios y la importación de bienes de consumo; una de las estaciones carboneras de Gran Bretaña, en tiempos de su hegemonía mundial en el paso del siglo XIX al siglo XX (Hidalgo, 2014, p. 14); el desarrollo industrial, mediante el caso representativo de Berisso y sus frigoríficos, con el arribo de las inversiones directas estadounidenses (Buxedas, 1983; Arrighi, 1999; Lluch, 2019); y la instalación de la destilería de Y.P.F., que conformará uno de los polos industriales petroquímicos más importantes de Argentina y de América Latina, recostándose, principalmente, sobre el municipio de Ensenada. Nuestro análisis comprende, entonces, un estudio de historia regional que se relaciona con las dinámicas globales a través del sistema portuario (Martner Peyrelongue, 1999). Por lo tanto, consideramos el carácter agrario del capitalismo en Argentina y sus vinculaciones con el desarrollo económico de la región rioplatense estableciendo diálogos entre lo rural, las energías -carbón e hidrocarburos- y la industria frigorífica. En virtud de ello, situamos al puerto de La Plata en el ámbito atlántico con el ánimo de darle un nuevo lugar en la historiografía platense al considerarlo desde una nueva perspectiva de análisis. En vistas de los estudios que nos preceden en esta temática, consideramos que aún resta dar cuenta de las características del funcionamiento de este puerto analizando, principalmente, el desempeño de las actividades económicas que 
dieron forma a su hinterland y a su foreland. En este sentido, planteamos estudiar el entramado económico que se estructura en torno al puerto teniendo en cuenta que forma parte de procesos regionales que exceden a una mirada meramente local. Nos interesa compartir este primer balance historiográfico de nuestra investigación dado que, a partir de nuestra búsqueda bibliográfica, podemos plantear que el puerto de La Plata ha sido un objeto de estudio poco abordado desde la Historia.

Desde nuestra disciplina, Lázzaro es quien dedicó una mayor atención a este objeto de estudio en artículos (1982a, 1982b) y trabajos colectivos (1982c), al mismo tiempo que lo incluyó en sus estudios dedicados al sistema portuario argentino $(1987,1992)$. Ahora bien, buscando más allá de sus investigaciones encontramos aportes de distintos autores y autoras, pero que sólo trabajaron de manera puntual, y sin continuidad, la cuestión del puerto platense. Vemos aquí una vacancia como campo de estudio de la Historia. En ese sentido, podemos contar el trabajo de Scoccia (1950), desde su rol como Administrador del Puerto de La Plata. Por otro lado, en el Primer Congreso Nacional de Historia de los puertos argentinos, que tuvo lugar en Buenos Aires, en 1985, hallamos los trabajos de Barreneche (1987) y de Arena de Tejedor y Games (1987). ${ }^{2}$ Finalmente, contamos con el trabajo de Scarfó (2000), aunque en realidad este se aboca a los puertos de Ensenada desde una mirada geográfica a través del tiempo en el largo plazo. ${ }^{3}$ Sobre este panorama, pudimos construir nuestro estado de la cuestión acudiendo a diferentes capítulos dedicados al puerto de La Plata en trabajos que enfocan al sistema portuario (Ortiz, 1943; García, 1976; Lázzaro, 1987) así como una multiplicidad de trabajos sobre la historia de La Plata que dedican parte de su atención al puerto, focalizando el interés en los momentos de su construcción, vinculados a la proyección y fundación de La Plata como nueva capital provincial en el año 1882 (Cygan, 1979; Bernard, 1982; Díaz, 1982; Lerange, 1982; Ortega, 1982; Rica, 1982; Tartarini, 1982; Barba, 1983, 1998; de Paula, 1987). ${ }^{5}$ Por otro lado, cabe mencionar que existen aportes muy significativos de la Geografía sobre el Puerto La Plata y su hinterland, desde un abordaje del territorio, pero que exceden nuestro período de estudio al estar concentrados en el período de nuestra historia reciente (Carut, 2008, 2010, 2014; Carut, Crivos, D'Amico, Arbide, Botana, 2015; Carut, D'Amico, Botana, Zabaljauregui, Valdez, Luciano, 2016; D'Amico, Gabriela; Carut, Ghetti, Arbide, Luciano, 2016; Carut, Crivos, Arbide, Stubbs, 2018; Carut, Arbide, Crivos, D'Amico, Ghetti, 2018; Carut et al, 2019). ${ }^{6}$

\section{El lugar del puerto de La Plata en los trabajos históricos Que abordaron la CUESTIÓN PORTUARIA}

A partir de Ortiz (1943) vemos al puerto de La Plata como una iniciativa política enmarcada en el período de constitución del sistema portuario argentino, planteada como continuidad de la actividad portuaria vinculada a Buenos Aires mediante la extensión de las vías ferroviarias que unieron las estaciones Casa Amarilla y Puerto Ensenada hacia finales de 1872 (Ortiz, 1943, p. 28). Este autor identifica, entonces, el proyecto de construir en La Plata el principal puerto de ultramar del país en virtud de su proximidad a las partes hondas del Río de la Plata. Es importante destacar que este trabajo ya señala el desfase entre el proyecto de puerto que impulsa el gobierno provincial y su concreción final, así como las dificultades financieras que afronta en su construcción y la competencia que representa la puesta en actividad de Puerto Madero, lo cual se manifestará con todo su peso en el traspaso a la Nación, en 1904, considerándose este hecho el resultado de su sumisión al puerto de Buenos Aires.

Scoccia (1950), aporta una visión diferente, planteada en términos de vivencia personal, en tanto ensenadense y Administrador de la Aduana y Puerto de La Plata. A partir de las referencias teóricas de "tratadistas de habla inglesa" (Scoccia, 1950, p. 11) propone ver al puerto de La Plata en función de la noción de "Puerto, en sentido amplio" contemplando las capacidades necesarias para las actividades de comercio fluvial, marítimo y aéreo, la industria y el movimiento de lanchas y ferrys (Scoccia, 1950, p. 14). Su mirada remite, inicialmente, al período colonial, pero está centrada en las primeras décadas del s. XX, 
dando especial atención al funcionamiento del puerto bajo la administración nacional. La etapa provincial previa, de construcción y puesta en funcionamiento -entre 1883 y 1904- es aludida en términos de "primeros pasos lentos, difíciles, inseguros" (Scoccia, 1950, p. 28). No obstante, no aparecen aquí las alusiones a una crisis ni fracaso inicial del Puerto, tal como apreciamos en la bibliografía posterior. Por el contrario, Scoccia describe cada parte del puerto y sus actividades correspondientes planteando un panorama de actividad y florecimiento. Este autor se distingue, también, por ser el único en considerar positivamente el traspaso del puerto de La Plata a la Nación, en función de otorgar mejores condiciones al movimiento de hacienda y carbón, así como a los talleres de la marina (Scoccia, 1950, p. 36). A cambio de ello, la Provincia delega parte de sus responsabilidades sobre los empréstitos tomados en el extranjero.

Para resaltar el carácter industrial que asume este puerto, Scoccia postula una suerte de genealogía económica. Toma como punto de partida a la concesión otorgada a Zavalla y Cía. para la construcción de un embarcadero de hacienda en pie en 1895, la cual se transformará en "una fábrica congeladora de carnes y conservas" desde 1900 (Scoccia, 1950, p. 39). Este itinerario continúa cuando, en abril de 1902, Zavalla y Cía. transfiere su concesión a Juan Tregidga, y éste -en julio de 1904- a The La Plata Cold Storage Company Limited, la cual, a partir de 1916 quedará registrada legalmente como Compañía Swift de La Plata Sociedad Anónima. En este recorrido, Scoccia apunta que hacia 1942, cuando vence el tiempo de concesión, Swift renuncia a la prórroga del contrato, pero negocia continuar operando bajo un permiso precario (1950, p. 41-42). El mismo periplo legal, aunque con actores distintos, recorrieron las tierras que en 1886 propusieron terraplenar los constructores del puerto, Lavalle y Médici, a cambio de su usufructo, el cual traspasaron a Luis Castells en 1887 y éste a la compañía Mercado de Frutos S. A. en 1888. Esta última demoró sus estatutos legales hasta julio de 1911, un mes antes de transferir sus derechos para la instalación del Frigorífico Armour S. A. Por otro lado, los mismos Lavalle y Médici aprovecharon una de las cláusulas suscritas en 1886, en el contrato de construcción del Puerto, para elevar los terrenos de la Isla Santiago a cambio de una parte de su propiedad, la cual dieron en concesión a la Compañía Muelles y Depósitos, que comenzó a explotar el servicio de cargas y descargas desde 1905 hasta 1945, cuando requirió la intervención del Ejecutivo Nacional a cargo de Juan D. Perón (Scoccia, 1950, p. 47). Nos preguntamos, entonces, en nuestra tesis, en qué medida las instalaciones del puerto de La Plata resultaron una carga económica difícil de sobrellevar o bien, un buen negocio inmobiliario del cual obtener beneficios como intermediario.

Esta visión de la historia del puerto de La Plata es reforzada con la enumeración de los contratos entre la Administración del Puerto con las compañías del Ferrocarril Oeste y Sud, hacia 1898 sumado a la autorización dada en 1909 a la Compañía General de FF. CC. de la Provincia de Buenos Aires para ligar sus vías con el acceso al Puerto de La Plata mediante una línea de trocha angosta (Scoccia, 1950, p. 52). Este tramo de crecimiento confluye con la naciente industria petrolera, cuando el General Mosconi, a cargo de Yacimientos Fiscales, impulsa la construcción de destilerías para procesar el crudo extraído en la Patagonia bajo la premisa "dejar de ganar, es perder" (Scoccia, 1950, p. 57). Entonces, desde comienzos de la década de 1920, el Puerto de La Plata comienza a estar en la órbita de la actividad hidrocarburífera. Scoccia resalta la eficiencia con el que se concretó la instalación de la destilería de YPF. No aparece nada semejante a dificultades de funcionamiento y operatividad portuaria que hayan podido afectar el desempeño de la destilería sino todo lo contrario; de las palabras de Scoccia se desprende el inicio de una rama de actividad en pleno crecimiento y potenciación de las instalaciones del puerto como, por ejemplo, la construcción de un taller para la reparación de buques. ${ }^{7}$

Entrada la década de 1980, Lázzaro propone un estudio más sistemático de los aspectos políticos, enfocados en la administración pública, que dan cuenta de la relevancia del proyecto portuario en la fundación de La Plata y, consecuentemente, en los intentos "federalistas" por competir con el predominio de Buenos Aires (Lázzaro, 1982a, 1982b, 1982c, 1987 y 1992). Desde esta mirada, que se aboca fundamentalmente a los primeros años del puerto -a partir de su proyección en torno a 1881 hasta su traspaso a la Nación en 1904 (Lázzaro, 1982a, pp. 291-292)- se destacan las dificultades financieras y comerciales que 
truncan las expectativas iniciales de constituir un polo comercial que no consigue dinamizar la región. Tal lectura se enriquece al poner en consideración el rol del Estado y del capital internacional en la conformación del sistema portuario argentino, lo cual posibilita plantear un campo de análisis signado por las tensiones que provocan las disputas políticas y económicas en el trasfondo del funcionamiento portuario (Lázzaro, 1987). Entonces, vemos sus trabajos también en sintonía con las preocupaciones de Ortiz (1943) recuperando el desafío de aportar desde la Historia un diagnóstico sobre los factores que subsumieron a este puerto en lo que caracteriza como una situación de letargo, enfocada principalmente en el período crítico de su funcionamiento entre 1897 y 1904 (1982a, 1982b, 1987, 1992). Es importante señalar que en sus últimos trabajos sobre el tema Lázzaro (1987 y 1992) vincula actores sociales concretos al fracaso del puerto de La Plata como: un Estado ineficiente en la construcción, mantenimiento y readecuación de las instalaciones portuarias; las elites terratenientes vinculadas con el comercio exportador que nuclean sus actividades en torno a Buenos Aires; y, los capitales extranjeros que monopolizan el sistema ferroviario, particularmente, FF. CC. Sud y FF. CC. Oeste.

En consecuencia, la autora nos propone una reconstrucción histórica de los aspectos que llevaron al puerto de La Plata a una situación de zozobra atendiendo, principalmente, a las decisiones políticas adoptadas por los gobernantes tanto a nivel provincial como nacional. Desde esta perspectiva, se enfatiza que la fundación de La Plata contuvo el proyecto de emplazar una capital para la provincia de Buenos Aires sustentada, fundamentalmente, en el desarrollo de un nodo económico en torno a la actividad comercial portuaria que fuera capaz de canalizar las exportaciones bonaerenses (Lázzaro, 1982a, 1982b, 1987). Así, su ubicación estuvo orientada por la opción de constituir una capital fluvial con una proyección portuaria de ultramar (Lázzaro, 1982a, 1982b, 1982c, 1987). Sin embargo, postula la autora, la competencia de Buenos Aires, "con su inalterada tendencia centralizadora" (Lázzaro, 1982a, p. 301; 1987), definirá los términos del fracaso del puerto platense.

Lázzaro nos permite recuperar el compendio de decisiones políticas involucradas en la puesta en funcionamiento del puerto de La Plata, dando cuenta, principalmente, de las tensiones, desavenencias e iniciativas erradas que este hecho político suscitó en su tiempo. ${ }^{8}$ Por otro lado, es reconstruida la "evolución comercial del puerto" señalando un curso ascendente del movimiento portuario, hasta 1896, y una caída a partir de 1897 que deviene en la crisis que justifica su traspaso a la nación, en octubre de 1904 (Lázzaro, 1982a, 1987). En este plano se enumeran algunos de los productos que conforman el tránsito de exportación, así como los obstáculos que presentan las compañías ferrocarrileras y la caída en las importaciones como consecuencia de la apertura del canal norte de puerto Madero, en 1898, representando este hecho el motivo por el cual compañías navieras de banderas italiana, francesa, española e inglesa dejan de operar en el puerto platense, lo cual se agrava hacia 1903 (Lázzaro, 1982b, 1987). En cuanto al período abierto con el traspaso a la esfera nacional de la administración del puerto de La Plata se abordan las intenciones manifestadas públicamente por los titulares del Ejecutivo Nacional, tal es el caso de Julio A. Roca, y del Ejecutivo Provincial, a cargo de Marcelino Ugarte. Los principales motivos esgrimidos fueron la decadencia del puerto de La Plata y su peso sobre el erario provincial, la necesidad de descongestionar el abarrotado puerto de Buenos Aires y su ampliación para constituirse en el principal puerto de aguas profundas frente al desarrollo del puerto de Montevideo (Lázzaro, 1982a, 1987, 1992). Es así como desde el gobierno nacional se plantea trasladar desde Buenos Aires a La Plata los depósitos de inflamables, embarcaderos de hacienda y los talleres de la Marina; mientras que, por su parte, las expectativas provinciales apuntan a vincular el puerto con "toda la zona agrícola de la provincia de Buenos Aires productora de trigo, maíz, lino (...)” (Lázzaro, 1982b, p. 10). ${ }^{9}$ De todas maneras, al mismo tiempo en que se señalan "momentos de absoluta paralización", entre 1905 y 1906 el Ministerio de Agricultura de la Nación tomó cartas en el asunto de la habilitación de instalaciones para la exportación de ganado en pie y de cereales (Lázzaro, 1982b, pp. 11-12; Lázzaro, 1987, p. 124-125). Inclusive, hacia 1904 tiene lugar la iniciativa de unir por ferrocarril a La Plata con Meridiano V, en la que intervienen Dirks y Dates, las casas bancarias de Otto Bemberg, Dreyfus y cia., y Emilio Erlanger y cia. (Lázzaro, 1987, 
p. 126). Finalmente, Lázzaro aporta una visión que señala las ventajas que ofrece La Plata para la llegada de capitales estadounidenses y británicos, durante la primera década del siglo XX, con la instalación de las plantas de Swift y Armour (1982b, 1982c). En ese sentido, al calor de la Primera Guerra Mundial, las facilidades de acceso a ultramar, así como la ventaja geográfica de hallarse más cerca de Europa en comparación con Australia, dan a nuestro puerto una actividad de exportación de carne bovina enfriada que representa, según los datos presentados por la autora, la mitad del total exportado a nivel nacional (Lázzaro, 1982b, p. 13). Esto se complementa con un mayor nivel de actividad de cabotaje, pero que, en la evaluación de la autora cuando compara datos del Anuario del movimiento portuario de los puertos de la República Argentina de 1944, "no hace más que constatar cuantitativamente la realidad cualitativa de la actividad del puerto: simple punto de tránsito para la introducción de mercaderías a Buenos Aires u otros centros de consumo" (Lázzaro, 1982b, p. 13). En este plano de actividades predominan el transporte de petróleo crudo y los productos derivados luego de su paso por la Destilería de Y. P. F., lo cual reafirma que "el rumbo del puerto de La Plata está marcado", ciertamente, desde tal perspectiva, en un sentido poco promisorio (Lázzaro, 1982b, p. 14). ${ }^{10}$

Barreneche (1987) recoge la situación de estancamiento en que se encuentra el puerto de La Plata al momento de escribir su trabajo. Desde esa inquietud indaga el período de gestión provincial entre 1880-1904, atendiendo a las complicaciones ocurridas en materia política y económica. En líneas generales, hay una mirada similar a la elaborada por Lázzaro, aunque podemos señalar aportes distintivos. Por ejemplo, en lo que refiere al entramado político, este autor liga la proyección del puerto a la negociación entre Dardo Rocha y Julio A. Roca en pos de encauzar la institucionalidad en crisis a finales del siglo XIX. Con Barreneche vemos, entonces, que la elección del sitio en Ensenada conjuga, además de las condiciones naturales propicias, un pacto político por sostener una distancia precavida entre los centros de decisión política y económica del momento. Tal argumentación implica, como vimos en Lázzaro, un trabajo minucioso por parte de Barreneche sobre las disposiciones políticas referidas al puerto y presenta, por su parte, una atención especial a los gastos, emisión de deuda y empréstitos tomados para la construcción del puerto. En ese sentido, Barreneche también identifica como dificultades los problemas presupuestarios para finalizar las obras del puerto; la deficiente conexión ferroviaria entre el puerto de La Plata y las zonas productivas de la provincia de Buenos Aires; la ausencia de un gran mercado consumidor en torno al puerto; y la inutilización de parte de sus muelles por la carencia en infraestructuras de almacenamiento (Barreneche, 1987, p. 36). Por otro lado, el desenvolvimiento de la actividad económica es graficado mediante cuadros comparativos que permiten observar, entre otros rasgos, la curva de ascenso en la actividad del puerto, desde su inauguración hasta 1897, con su posterior declive hacia $1904 .{ }^{11}$ Asimismo, el panorama crítico del puerto platense en los años 1898-1904 es visto por Barreneche en sintonía con las apreciaciones que vimos en Lázzaro. ${ }^{12}$ Barreneche aborda el traspaso del puerto de La Plata a la administración nacional concretando, finalmente, su carácter auxiliar al ser incorporado al de la Capital Federal (1987, p. 46).

Más allá de esta coincidencia general con varias de las perspectivas que conforman nuestro estado de la cuestión, en sus conclusiones, Barreneche aporta una mirada propia en virtud de los datos que analiza. Este autor plantea que durante todo el período de gestión provincial el puerto de La Plata funciona de manera subsidiaria del de Buenos Aires, más allá de los buenos años que tiene entre 1891 y 1896 . Asimismo, establece como carácter diferencial que cuando cae la actividad del movimiento portuario también hay una decadencia de los principales rubros que circulan por el puerto, tales son los cueros, tasajo y hacienda en pie, y no surgen otros rubros que los reemplacen. Esta perspectiva nos interesa particularmente porque vemos en ella ciertos indicios de especialización en los rubros que mueve el puerto platense lo cual nos conduce a plantear la necesidad de ampliar el marco de análisis temporal y espacial para poder comprender las dinámicas sociales y productivas comprendidas en nuestro caso de estudio. En este sentido, vemos que los aspectos cualitativos de la cartera de productos que circulan por el puerto platense prácticamente no han sido considerados.

Arena de Tejedor y Games (1987), nos aportan una mirada particular sobre un tema puntual que prácticamente no había sido tratado: los factores financieros en la construcción del puerto de La Plata. 
Parten de considerar que el desarrollo económico de la segunda mitad del siglo XIX tuvo como elementos básicos el movimiento internacional de capitales, las corrientes migratorias y la expansión del comercio mundial, para caracterizar -en ese contexto histórico- a la gobernación de Dardo Rocha con una clara política de endeudamiento externo. Consideramos de importancia esta perspectiva ya que reflexiona sobre las condiciones financieras favorables que presenta la provincia a principios de la década de 1880 pero que, sin embargo, prontamente queda en una situación que sobrepasa su capacidad económica. Es interesante recuperar, por otro lado, que una parte importante de las fuentes trabajadas son la correspondencia de Dardo Rocha con diferentes actores de aquel momento. Mediante estos documentos, junto a otros, como artículos de prensa y documentos del Ministerio de Obras Públicas de la Pcia. de Bs. As., las autoras dan cuenta, minuciosamente, de las características de los títulos de deuda pública emitidos y su colocación como empréstitos. Nos interesa remarcar que los prestamistas de Londres y París se acercan interesados ante las iniciativas provinciales y que la negociación se realiza con Bemberg y Heimendalk en tanto representantes del sindicato de banqueros franceses, presididos por Morton Rosse y Cia., quienes financiarán tres cuartas partes del empréstito, mientras que la firma Stern-Brothers aporta la parte restante, aclaran las autoras, "no obstante no aparecer la firma de los últimos en el contrato" (Arena de Tejedor y Games, 1987, p. 17). Pese a "conflictos internos entre las casas prestamistas" (Arena de Tejedor y Games, 1987, p. 17), los empréstitos se concretan, y el aporte de estas autoras nos deja abierta la pregunta sobre quiénes son los actores económicos que toman los títulos de deuda con el propósito de discernir a favor de qué intereses quedan hipotecadas las obras y el producido del puerto platense. Vemos en ese sentido la posibilidad de avanzar en nuestro planteo de situar al puerto platense en el sistema atlántico sumando al estudio de su participación en las redes del comercio mundial aportar, también, a clarificar cuál ha sido su lugar en el movimiento de capitales internacionales. ${ }^{13}$

\section{NUESTRO BALANCE COMO PUNTO DE PARTIDA}

Esta primera etapa de nuestra investigación nos permite plantear un balance inicial desde el cual vislumbrar un campo de estudio promisorio respecto del puerto de La Plata tanto para las Ciencias Sociales en general como para la Historia en particular.

Con Ortiz (1943) pudimos apreciar el desfase ocurrido entre los proyectos impulsados desde el Estado en torno a la proyección del puerto y su concreción final. Esta perspectiva de análisis continúa en los distintos trabajos de Lázzaro (1982a, 1982b, 1982c, 1987, 1992), Barreneche (1987), Arena de Tejedor y Games (1987),Scarfó (2000) y el resto de la bibliografía referida particularmente a la historia de la ciudad de La Plata (Cygan, 1979; Bernard, 1982; Díaz, 1982; Lerange, 1982; Ortega, 1982; Rica, 1982; Tartarini, 1982; Morosi, 1983; Barba, 1983, 1998; de Paula, 1987). En este aspecto, predomina una mirada focalizada, principalmente, en el período 1883-1904. Por su parte, Scoccia (1950) se diferencia en este punto al considerar prioritariamente el período portuario abierto con el traspaso a la Nación a partir de 1904. Podemos señalar, en este orden, que los distintos períodos de tiempo considerados también implican comprensiones diferentes sobre los procesos económicos que atañen a nuestro puerto. Por ejemplo, quienes observan el período de gestión provincial concurren en señalar con énfasis el período crítico que tuvo lugar hacia 1897 y desencadena el traspaso del puerto en 1904 como una confirmación del fracaso. Por otro lado, Scoccia (1950) describe para ese período, particularmente en torno a 1898 , una serie de transacciones privadas que anteceden la instalación de la industria frigorífica. Desde su mirada, rescata el carácter industrial que comienza a tener el puerto -énfasis que no aparece como un valor positivo en otros trabajos- denotando una situación bastante alejada del aletargamiento o fracaso con que ha solido verse al puerto de La Plata. Ciertamente, esta mirada está dada desde una época particular y seguramente sus énfasis deben considerarse en ese sentido, pero tal apreciación no es menos acertada para el resto de los análisis sobre el puerto, inclusive el nuestro, claro. Lo que nos interesa remarcar a partir de esta contraposición remite a la pertinencia de 
proponer una ampliación del espectro temporal para sopesar los rasgos y profundidad de una primera etapa, problemática, por cierto, de funcionamiento del puerto, delimitable hacia finales del siglo XIX.

Por otro lado, a partir de Lázzaro (1982a, 1982b, 1982c, 1987, 1992) y Barreneche (1987) contamos con una base muy importante sobre a las actuaciones de gobierno que intervinieron en la construcción y puesta en funcionamiento del puerto, que en gran medida se replica en el resto de la bibliografía. Retomando estos aportes es posible comenzar a plantear un estudio que profundice en los procesos inherentes a las políticas públicas para así establecer los actores sociales intervinientes, sus tensiones e intereses ubicados en su contexto histórico.

Asimismo, hemos notado la reiteración sobre dos cuestiones problemáticas que se relacionan con el fracaso del proyecto inicial del puerto de La Plata y la competencia que representa el puerto de Buenos Aires (Ortiz, 1943; García, 1976; Cygan, 1979; Lázzaro, 1982a, 1982b, 1982c, 1987, 1992; Bernard, 1982; Díaz, 1982; Lerange, 1982; Ortega, 1982; Rica, 1982; Tartarini, 1982; Morosi, 1983; Barba. 1983, 1998; Arena de Tejedor y Games, 1987; Barreneche, 1987; de Paula, 1987; Scarfó, 2000). Sin perder de vista la importancia del aporte que el conjunto de estos trabajos representa, el posible denotar cierta carencia explicativa del funcionamiento específico del puerto y de su rol dentro del sistema portuario argentino. Por ejemplo, no hay aportes significativos sobre los rasgos económicos cualitativos de qué se transportaba mediante el puerto, cómo se realizaron las operaciones comerciales, con qué tecnologías, quiénes operaban, en qué condiciones, etc., ni mucho menos cuál fue su rol dentro del conjunto de los puertos del río de la Plata. En este sentido, nos parece importante plantear la importancia de situar al puerto de La Plata dentro del esquema de circulación atlántica; $y$, en este aspecto, determinar qué lugar ocupa dentro del sistema de los puertos argentinos para poder aportar cuál ha sido su incidencia en el desarrollo regional. De aquí surge la observación con respecto a la vacancia en el conocimiento actual sobre la conformación, forma y tipo del hinterland y el foreland del puerto platense, tal como lo ha hecho Mateo (2014) para el caso de Quequén, lo cual puede ser un aporte para complejizar los análisis que abordan las cuestiones relativas a su funcionamiento. Por ejemplo, a partir del trabajo de Hidalgo (2014) sobre el puerto de Dakar (Senegal) hallamos al puerto La Plata, a través del transporte y depósitos de carbón, como un engranaje importante del imperialismo británico a finales del siglo XIX. En otro orden, este tipo de vinculaciones también nos abre un nuevo panorama de interrogantes sobre las políticas de endeudamiento externo y la llegada de capitales invertidos en infraestructura ferrocarrilera y portuaria, mediante las cuales podremos caracterizar los sujetos y agentes económicos que transitan nuestro puerto, que, como vimos en Arena de Tejedor y Games (1987), no parecen haber estado exentos de conflictos, en un contexto mundial marcado por el declive de la hegemonía británica y el ascenso de EE. UU. (Arrighi, 1998).

Entonces, cabe preguntarse y comenzar a desarrollar ¿en qué medida resultó un fracaso el puerto de La Plata? ¿Cómo fue qué, de su crisis inicial en torno a comienzos del siglo XX, luego pasará a contener una diversidad de movimientos tales como la exportación de cereales y carne al tiempo que aumenta el cabotaje vinculado a la actividad hidrocarburífera y la importación de insumos industriales y la construcción? La pregunta sobre el fracaso, y su respuesta en modo de causa-efecto ¿es contemplativa de la complejidad que encierra un puerto, en tanto entramado económico, social, político?

En términos generales, vemos que aún resta determinar para una historia del puerto de La Plata cuáles son las dinámicas en las que se mueven los actores y sujetos que dieron forma a nuestra región. Nos interesa abrir interrogantes que refieren a otros planos de las decisiones políticas que marcaron el derrotero de la actividad portuaria, no sólo su esfera institucional, para prestar mayor atención al trasfondo sociohistórico en el que tienen lugar. Dejamos abiertas, entonces, algunas preguntas a desentrañar ¿qué factores entran en juego en las reconfiguraciones socio-productivas de esta región fluvial a partir de la decisión política de construir el Puerto La Plata en 1883 ? ¿Cómo es que se producen las sucesivas readecuaciones productivas de la economía en la zona portuaria? ¿Es posible realizar una mirada desde la perspectiva de las políticas del desarrollo? ¿Qué 
actores y sujetos sociales intervienen? ¿Quiénes son? ¿Quiénes y de qué se benefician? ¿Quiénes ganan y pierden?

\section{BibLIOgRAFÍA}

Arena de Tejedor, F. y Games, A. B. (1987). Factores financieros de la construcción del puerto de La Plata y su posterior evolución hasta 1900. Trabajo presentado en Primer Congreso Nacional de Historia de los Puertos Argentinos, celebrado en Buenos Aires, noviembre de 1985.

Arrighi, G. (1999). El largo siglo XX. España: Ediciones Akal.

Asnaghi, C. A. (2004). Ensenada: una lección de Historia. Argentina: 2da. Edición, Municipalidad de Ensenada.

Barba, F. E. (1983). El momento histórico de la fundación de La Plata. En Morosi, J. A. y Terán, F. (Dir.), La Plata. Ciudad nueva, ciudad antigua. Historia, forma y estructura de un espacio urbano singular (pp. 17-26). La Plata: Universidad Nacional de La Plata e Instituto de Estudios de Administración Local (España).

Barba, F. E. (1998). La Plata en sus primeros años de vida, Revista Museo, 49-54. La Plata.

Barreneche, O. (1987). Política y realidad económica del puerto La Plata 1880-1904. Trabajo presentado en Primer Congreso Nacional de Historia de los Puertos Argentinos, celebrado en Buenos Aires, noviembre de 1985.

Bernard, T. D. (1982). El momento histórico de la fundación de La Plata, en Lerange, C. (Dir), La Plata Ciudad Milagro (pp. 21-30). La Plata: Corregidor.

Buxedas, M. (1983). La industria frigorifica en el Río de la Plata (1959-1977). Bs. As.: CLACSO.

Carut, C. (2008) La metropolización costera. Un juego dialéctico de los lugares: Entre la inserción y su aislamiento en la nueva metropolización (En línea). Trabajo presentado en II Jornadas del Doctorado en Geografía, 14 de mayo de 2008, La Plata, Argentina. Disponible en: http://www.memoria.fahce.unlp.edu.ar/trab_eventos/ev.7 48/ev.748.pdf

Carut, Claudia (2010) Categorías de análisis para un abordaje político del espacio estuarial metropolitano en la década de los 90: Región Metropolitana de Buenos Aires (En línea). Trabajo presentado en III Jornadas del Doctorado en 6Geografía, 29 y 30 de septiembre de 2010, La Plata, Argentina. Disponible en: http://www.memoria.fahce .unlp.edu.ar/trab_eventos/ev.1495/ev.1495.pdf

Carut, C. (2014) La metropolización costera de la Región Metropolitana de Buenos Aires: Un juego dialéctico de los lugares: Entre la inserción y su aislamiento en la nueva metropolización de la década de los 90 (Tesis de posgrado). Presentada en Universidad Nacional de La Plata. Facultad de Humanidades y Ciencias de la Educación para optar al grado de Doctora en Geografía. Disponible en: http://www.memoria.fahce.unlp.edu.ar/tesis/te.986/t e.986.pdf

Carut, C.; Crivos, M.; D'Amico, G.; Arbide, D.; Botana, M. (2015). El puerto y su hinterland: análisis de las transformaciones generadas a partir de la década de los 90 en el área sur del estuario del Río de La Plata. XVII Jornadas de Geografía de la UNLP, 11 y 12 de noviembre de 2015, La Plata, Argentina. EN: XVII Jornadas (2015). La Plata: UNLP-FaHCE. Disponible en: http://www.memoria.fahce.unlp.edu.ar/trab_eventos/ev.94 47/ev.9447.pdf

Carut, Claudia (2015) La invisibilidad neoliberal y la identidad de los territorios costeros : El caso de las transformaciones de la costa vitivinícola de Berisso. Mundo Agrario, 16 (31). Disponible en: http://www.mem oria.fahce.unlp.edu.ar/art_revistas/pr.6739/pr.6739.pdf

Carut, C., D'Amico, G., Botana, M. I., Zabaljauregui, M., Valdez, A., Luciano, M. (2016). Las herencias territoriales como reflejos de los cambios portuarios: El caso del Puerto La Plata, Buenos Aires (Argentina). En: Geografias en diálogo: aportes para la reflexión. Tandil: Universidad Nacional del Centro de la Provincia de Buenos Aires. p. 289-296. Disponible en: http://www.memoria.fahce.unlp.edu.ar/libros/pm.802/pm.802.pdf

Carut, C.; Crivos, M.; Arbide, D.; Stubbs, E. (2018). El abordaje transdiciplinar en los estudios portuarios: el Puerto La Plata y la Isla Paulino, Berisso. Argentina. I Jornadas Platenses de Geografía, 17 al 19 de octubre de 2018, La Plata, Argentina. EN: Actas. La Plata: Universidad Nacional de La Plata. Facultad de Humanidades y Ciencias 
de la Educación. Departamento de Geografía. Disponible en: http://www.memoria.fahce.unlp.edu.ar/trab_ev entos/ev.11248/ev.11248.pdf

Carut, C.; Arbide, D.; Crivos, M.; D'Amico, G.; Ghetti, G. (2018). Las herencias territoriales como forma de comprender el espacio del Puerto La Plata. I Jornadas Nacionales de Geografía, 18 al 20 de abril de 2018, Mar del Plata, Argentina. EN: Geografía, espacio y sociedad en los debates actuales. Mar del Plata: Universidad Nacional de Mar del Plata, 387-399. Disponible en: http://www.memoria.fahce.unlp.edu.ar/trab_eventos/ev.7138/ev.7 138.pdf

Carut, C.; Crivos, M.; D'Amico, G.; Toledo, M. (2019). Continuidad y cambio en las trayectorias productivas de la Isla Paulino desde 1936 a la actualidad (Berisso, Argentina). XXI Jornadas de Geografía de la UNLP, 9 al 11 de octubre de 2019, Ensenada, Argentina. Construyendo una Geografía Crítica y Transformadora: En defensa de la Ciencia y la Universidad Pública. EN: Actas. Ensenada: Universidad Nacional de La Plata. Facultad de Humanidades y Ciencias de la Educación. Disponible en: http://www.memoria.fahce.unlp.edu.ar/trab_evento s/ev.13516/ev.13516.pdf

Cygan, G. M. (1979). La ciudad de La Plata en su centenario. Fundamentos para una nueva Argentina. La Plata (sin editorial).

D'Amico, G.; Carut, C.; Ghetti, G.; Arbide, D.; Luciano, M. (2016) Transformaciones de las costas estuariales: El caso del Puerto La Plata y la Isla Paulino (Argentina). Tiempo y Espacio, (35): 150-168. Disponible en: http://www .memoria.fahce.unlp.edu.ar/art_revistas/pr.7748/pr.7748.pdf

de Paula, Alberto S. J. (1987) La Ciudad de La Plata. Sus tierras y su arquitectura. La Plata: Banco de la Provincia de Buenos Aires.

Díaz, B. (Dir.) (1982). La Plata, una Obra de Arte 1882-1982. La Plata: Universidad Nacional de La Plata.

García, D. (1976). El desarrollo portuario argentino. Tesis Doctoral. Facultad de Ciencias Económicas, UBA.

Hidalgo, D. C. (2015). Las puertas del Imperio. Análisis del sistema portuario de Senegal: jerarquía, centralidad y complementariedad en un contexto colonial, 1839-1910. Investigaciones de Historia Económica-Economic History Research, 11(2), 91-102.

Lázzaro, S. B. (1982a). El Puerto de La Plata: Aspectos de su evolución histórica (1882-1904). En Investigaciones y Ensayos, 32, 291-322. Academia Nacional de la Historia, Buenos Aires.

Lázzaro, S. B. (1982b). El proceso histórico del puerto de La Plata 1882-1982. Revista de La Bolsa de Comercio de La Plata, 1, 7-15.

Lázzaro, S. B. (1987). Historia de los puertos argentinos 1880-1914. Tesis de doctorado. Facultad de Humanidades y Ciencias de la Educación, Universidad Nacional de La Plata.

Lázzaro, S. B. (1992). Estado, capital extranjero y sistema portuario argentino (Vol. 2). Buenos Aires: CEAL.

Lerange, C. (1982). La Plata, ciudad milagro. Buenos Aires: Ed. Corregidor.

Lluch, A. (2019). Las empresas frigoríficas en Argentina: estrategias empresariales y cambios en el sector industrial (1882-1930). America Latina en la Historia Económica, 26(2), 1-23.

Martner Peyrelongue, C. (1999). El puerto y la vinculación entre lo local y lo global, EURE, XXV(75), 103-120.

Mateo, J. (2014). El impacto de un nuevo puerto: la construcción de su hinterland y de su foreland. Puerto Quequén, provincia de Buenos Aires entre 1921 y 1932. Mundo Agrario, 15(29). Recuperado a partir de https://www.m undoagrario.unlp.edu.ar/article/view/5356

Ortega, E. (1982). Último momento poblacional argentino. Inicios de La Plata como capital bonaerense, Revista de la Universidad, 28, 69-80. La Plata.

Ortiz, R. (1943). Valor económico de los puertos argentinos. Buenos Aires: Editorial Losada.

Prieto, S.; Banzato, G. (2017). Los vecinos del Barrio El Dique y los problemas ambientales: Primeros avances hacia una agenda de gestión con Inteligencia Territorial. En Saquet, M. e Alves, E. (Org.). Processos de cooperaçao e solidariedade na América Latina. Rio de Janeiro: Editora Consequência. Disponible en: http://www.memoria. fahce.unlp.edu.ar/libros/pm.510/pm.510.pdf 
Rica, J. A. (1982). Apuntes en el 1er centenario de La Plata. En Lerange, C. (Dir), La Plata Ciudad Milagro (pp. 31-36). La Plata: Ed. Corregidor.

Scarfó, G. (2000). Ensenada y sus puertos. En: Criado de Val, M. (Ed.). Caminería hispánica: actas del IV congreso Internacional. Guadalajara, España.

Scoccia, J. (1950). Puerto La Plata: definición, origen, evolución, realidad. La Plata, Argentina.

Tartarini, J. D. (1982). La acción profesional en la fundación de La Plata. La Plata: Consejo Profesional de la Ingeniería de la Provincia de Buenos Aires.

\section{Notas}

1 A partir de entonces, con el desarrollo de la actividad petrolera, la economía portuaria cambia significativamente y merece una atención especial que nos proponemos abordar en nuestra formación doctoral.

2 Agradecemos, especialmente, a Osvaldo Barreneche por facilitarnos la ubicación de su trabajo, realizado durante su formación de grado. En tal Congreso, las autoras Arena de Tejedor y Games presentaron también otro trabajo, "La Ensenada y su puerto; antecedentes históricos y construcción”. Así como Lázzaro, por su parte, participó con "El puerto de aguas profundas en la provincia de Buenos Aires: un antecedente histórico". En función de nuestro tema, decidimos no incluirlos en nuestro estado de la cuestión, pero nos parece oportuno dar cuenta de tales participaciones.

3 Es preciso mencionar que existe bibliografía especializada sobre la historia del Río de la Plata en la etapa virreinal. En nuestra casa de estudios, contamos con las líneas de investigación elaboradas por Fernando Jumar (2001, 2002, 2004, 2007, 2008, 2009) y Emir Reitano (2003, 2015, 2016), con sus respectivos equipos de investigación. Por su parte, queremos mencionar el trabajo pionero de Guillermina Sors de Tricerri (1933) abocado al período 1727-1810, el cual ha guiado muchas de las lecturas y elaboraciones de historiadores locales.

4 En este balance no nos explayaremos sobre el trabajo de García (1976) por tratarse de una tesis doctoral en Economía. Cabe señalar que sus apreciaciones son similares a las que reseñamos en Ortiz (1943).

5 Este grupo de trabajos, así como Scarfó (2000), se encuentra desarrollados con mayor extensión en el estado de la cuestión de nuestra tesis. Por otro lado, como puede observarse en los años de publicación, varias de estas obras forman parte de ediciones a propósito del centenario de la ciudad de La Plata; para profundizar en su caracterización recomendamos los estudios realizados por Badenes (2012, 2015).

6 Esta perspectiva de análisis continúa su desarrollo en el Proyecto de Investigación: Carut, Claudia B. (Dir.) (2019) Transformaciones territoriales de los puertos estuariales de Buenos Aires a partir de la reconversión portuaria de la década de los 90. El caso del Puerto La Plata / H886.

7 Este clima de resurgimiento del puerto de La Plata, con un perfil productivo industrial, podemos corroborarlo en la comunicación parlamentaria elevada por Gabriel del Mazo, junto a Ricardo Balbín y Emilio del Carril, en junio de 1949.

8 En el Capítulo 1 y estado de la cuestión de nuestra tesis retomamos el minucioso trabajo que presenta Lázzaro (1982a, 1982b, 1987) sobre los debates parlamentarios y mensajes de gobernadores donde se reconstruyen tales tensiones y desavenencias políticas.

9 En el Capítulo 2 de nuestra tesis buscamos problematizar los intereses y objetivos contrapuestos en las iniciativas que podemos leer recurriendo a los estudios de las políticas públicas, retomando el enfoque planteado por Oszlak y O’Donnell (1981).

10 A partir de entonces, el perfil portuario ligado a los hidrocarburos se consolida y profundiza, principalmente, a partir de 1956, a lo cual se suman las industrias petroquímicas y siderúrgicas, como el caso de Propulsora que construye su propio puerto en 1967.

11 En el Capítulo 3 de nuestra tesis retomamos los aportes que Barreneche realiza sobre el desenvolvimiento de la actividad económica del puerto de La Plata ya que nos acerca un conjunto de datos sistematizados que en el corpus bibliográfico que hemos relevado está disperso o no aparece. En su trabajo sistematiza fuentes muy importantes como la Memoria del Ministerio de Obras Públicas de la Pcia. de Buenos Aires, para los años 1894a 1896; Memorias descriptivas sobre el proyecto de ensanche del Puerto La Plata, publicado por el Ministerio de Obras Públicas bonaerense en 1898; las Memorias de la Administración General del Puerto La Plata, para los años 1897-1903; Memorias del Ministerio de Hacienda de la Nación, año 1891; Anuario de la Dirección General de Estadística, años 1900 y 1902;el trabajo elaborado por el Ing. Baldassari, en 1925, en la Dirección General de Navegación y Puertos. "Vías Navegables y Puertos de la República Argentina”, entre otras.

12 Es dable agregar que Barreneche recupera un conjunto de datos dispuestos a comparar al puerto de La Plata con el de Buenos Aires en varios aspectos: sus porcentajes de importación y exportación, en los decenios 1890-1900 y 1900-1910; los porcentajes de participación en el valor total de exportaciones e importaciones del país, para los años 1891-1902; 
y los costos por kilómetros recorridos de ferrocarril para la lana, maíz, trigo y lino, provenientes de Luján, Mercedes, Chivilcoy y 9 de Julio. En cada grupo de variables la diferencia está a favor de Buenos Aires, lo cual contribuye a sostener la tesis de su superioridad en detrimento del puerto de La Plata.

13 Luego de tratar la faz financiera, las autoras pasan a considerar la cuestión de las tierras en torno al puerto desde una mirada centrada en las disposiciones administrativas que atañen a lo jurisdiccional y los derechos de propiedad de estas debido al peso de los juicios que el procedimiento de expropiaciones generó. En este aspecto es interesante tomar nota del impacto que tuvo el inicio de la construcción del puerto en la valorización de las tierras aledañas, pero que a medida que se desenvolvieron sus problemas de funcionamiento esa tendencia retornó como una pérdida que pesó sobre el Banco Hipotecario de la Provincia. Por otro lado, al finalizar su trabajo las autoras atienden al desarrollo portuario hasta 1904, en el mismo sentido analítico que los trabajos mencionados anteriormente. Se enfatiza, de esta manera, una caracterización del puerto de La Plata como proyecto inconcluso lindante al fracaso.

\section{BY-NC-SA}

